\title{
$q$-Series congruences involving statistical mechanics partition functions in regime III and IV of Baxter's solution of the hard-hexagon model
}

\author{
Mircea Merca \\ Department of Mathematics, University of Craiova, Romania \\ Academy of Romanian Scientists, Bucharest, Romania \\ mircea.merca@profinfo.edu.ro
}

\begin{abstract}
For each $s \in\{2,4\}$, the generating function of $R_{s}(n)$, the number of partitions of $n$ into odd parts or congruent to $0, \pm s(\bmod 10)$, arises naturally in regime III of Rodney Baxter's solution of the hard-hexagon model of statistical mechanics. For each $s \in\{1,3\}$, the generating function of $R_{s}^{*}(n)$, the number of partitions of $n$ into parts not congruent to $0, \pm s$ $(\bmod 10)$ and $10-2 s(\bmod 20)$, arises naturally in regime IV of Rodney Baxter's solution of the hard-hexagon model of statistical mechanics. In this paper, we investigate the parity of $R_{s}(n)$ and $R_{s}^{*}(n)$, providing new parity results involving sums of partition numbers $p(n)$ and squares in arithmetic progressions.
\end{abstract}

Keywords: partitions, parity, hard-hexagon model

MSC 2010: 11P81, 11P83, 05A17, 05A19

\section{Introduction}

A partition of a positive integer $n$ is a sequence of positive integers whose sum is $n$. The order of the summands is unimportant when writing the partitions of $n$, but for consistency, a partition of $n$ will be written with the summands in a nonincreasing order [2. As usual, we denote by $p(n)$ the number of the partitions of $n$. For example, we have $p(5)=7$ because the partitions of 5 are given as:

$5,4+1,3+2,3+1+1,2+2+1,2+1+1+1,1+1+1+1+1$.

The fastest algorithms for enumerating all the partitions of an integer have recently been presented by Merca 8,9 . 
The partition function $p(n)$ may be defined by the generating function

$$
\sum_{n=0}^{\infty} p(n) q^{n}=\frac{1}{(q ; q)_{\infty}}
$$

Here and throughout this paper, we use the following customary $q$-series notation:

$$
(a ; q)_{0}=1, \quad(a ; q)_{n}=\prod_{k=0}^{n-1}\left(1-a q^{k}\right), \quad(a ; q)_{\infty}=\prod_{k=0}^{\infty}\left(1-a q^{k}\right) .
$$

Because the infinite product $(a ; q)_{\infty}$ diverges when $a \neq 0$ and $|q| \geqslant 1$, whenever $(a ; q)_{\infty}$ appears in a formula, we shall assume $|q|<1$ and we shall use the compact notation

$$
\left(a_{1}, a_{2} \ldots, a_{n} ; q\right)_{\infty}=\left(a_{1} ; q\right)_{\infty}\left(a_{2} ; q\right)_{\infty} \cdots\left(a_{n} ; q\right)_{\infty} .
$$

For $s \in\{2,4\}$, we denote by $R_{s}(n)$ the number of partitions of $n$ into odd parts or congruent to $0, \pm s(\bmod 10)$. Elementary techniques in the theory of partitions give the following generating function for $R_{s}(n)$ :

$$
\sum_{n=0}^{\infty} R_{s}(n) q^{n}=\frac{1}{\left(q ; q^{2}\right)_{\infty}\left(q^{s}, q^{10-s} ; q^{10}\right)_{\infty}} .
$$

The generating function for $R_{s}(n)$ arises naturally in regime III of Rodney Baxter's solution of the hard-hexagon model of statistical mechanics, appearing in the following $q$-identities of Rogers [1, 3]:

$$
\sum_{n=0}^{\infty} \frac{(-q ; q)_{n} q^{n(3 n+1) / 2}}{(q ; q)_{2 n+1}}=\frac{G\left(q^{2}\right)}{\left(q ; q^{2}\right)_{\infty}}
$$

and

$$
\sum_{n=0}^{\infty} \frac{(-q ; q)_{n} q^{3 n(n+1) / 2}}{(q ; q)_{2 n+1}}=\frac{H\left(q^{2}\right)}{\left(q ; q^{2}\right)_{\infty}}
$$

where

$$
G(q):=\sum_{n=0}^{\infty} \frac{q^{n^{2}}}{(q ; q)_{n}}=\frac{1}{\left(q, q^{4} ; q^{5}\right)_{\infty}}
$$

and

$$
H(q):=\sum_{n=0}^{\infty} \frac{q^{n^{2}+n}}{(q ; q)_{n}}=\frac{1}{\left(q^{2}, q^{3} ; q^{5}\right)_{\infty}}
$$

are the Rogers-Ramanujan functions.

For $s \in\{1,3\}$, we denote by $R_{s}^{*}(n)$ the number of partitions of $n$ into parts not congruent to $0, \pm s(\bmod 10)$ and $10-2 s(\bmod 20)$. Elementary techniques in the theory of partitions give the following generating function for $R_{s}^{*}(n)$ :

$$
\sum_{n=0}^{\infty} R_{s}^{*}(n) q^{n}=\frac{\left(q^{s}, q^{10-s}, q^{10} ; q^{10}\right)_{\infty}\left(q^{10-2 s}, q^{10+2 s} ; q^{20}\right)_{\infty}}{(q ; q)_{\infty}} .
$$


The generating function for $R_{s}^{*}(n)$ arises naturally in regime IV of Rodney Baxter's solution of the hard-hexagon model of statistical mechanics, appearing in the following $q$-identities of Rogers [1, 3]:

$$
\sum_{n=0}^{\infty} \frac{q^{n(n+1)}}{(q ; q)_{2 n}}=\frac{\left(q, q^{9}, q^{10} ; q^{10}\right)_{\infty}\left(q^{8}, q^{12} ; q^{20}\right)_{\infty}}{(q ; q)_{\infty}}
$$

and

$$
\sum_{n=0}^{\infty} \frac{q^{n(n+1)}}{(q ; q)_{2 n+1}}=\frac{\left(q^{3}, q^{7}, q^{10} ; q^{10}\right)_{\infty}\left(q^{4}, q^{16} ; q^{20}\right)_{\infty}}{(q ; q)_{\infty}} .
$$

In this paper, we shall provide the following $q$-series congruences.

Theorem 1.1. Let $s$ be a positive integer.

1. For $s \in\{2,4\}$,

$$
\sum_{n=0}^{\infty} \frac{(-q ; q)_{n} q^{n(3 n+s-1) / 2}}{(q ; q)_{2 n+1}} \equiv \sum_{120 n+(3 s-5)^{2}} q_{\text {square }} q^{n} \quad(\bmod 2) .
$$

2. For $s \in\{1,3\}$,

$$
\sum_{n=0}^{\infty} \frac{q^{n(n+1)}}{(q ; q)_{2 n+(s-1) / 2}} \equiv \sum_{40 n+s^{2} \text { square }} q^{n} \quad(\bmod 2) .
$$

As a consequence of Theorem 1.1 we immediately deduce the following parity result involving sums of partition numbers $p(n)$ and squares in arithmetic progressions.

Corollary 1.2. Let $n$ be a nonnegative integer.

1. For $s \in\{2,4\}$,

$$
\sum_{20 k+(s-1)^{2}} p(n-k) \equiv 1 \quad(\bmod 2)
$$

if and only if $120 n+(3 s-5)^{2}$ is a square.

2. For $s \in\{1,3\}$,

$$
\sum_{15 k+(s+1)^{2} / 4} p(n-k) \equiv 1 \quad(\bmod 2)
$$

if and only if $40 n+s^{2}$ is a square. 
Questions regarding the parity of sums of partition numbers for square values in given arithmetic progressions have been studied recently [6]. As we can see in [6], the cases $s=1$ and $s=2$ of Corollary 1.2 are known. The following conjecture is also known: the statement

$$
\sum_{a k+1 \text { square }} p(n-k) \equiv 1 \quad(\bmod 2) \quad \text { if and only if } \quad b n+1 \text { is a square. }
$$

is true if and only if

$$
(a, b) \in S:=\{(6,8),(8,12),(12,24),(15,40),(16,48),(20,120),(21,168)\} .
$$

This paper opens new possibilities for research in this area.

The organization of the paper is as follows. We will first prove Theorem 1.1 in Section 2 considering a truncated theta identity of Gauss 4 . In Section 3 . we will provide a proof of Corollary 1.2 considering a decomposition of $R_{s}(n)$ in terms of Euler partition function $p(n)$. In Section 4, we will introduce new open problems involving the partition function $R_{s}(n), s \in\{2,4\}$ and $R_{s}^{*}(n)$, $s \in\{1,3\}$.

\section{Proof of Theorem 1.1}

Watson's quintuple product identity [5, 10] states that

$$
\sum_{n=-\infty}^{\infty} z^{3 n} q^{n(3 n-1) / 2}\left(1-z q^{n}\right)=(q, z, q / z ; q)_{\infty}\left(q z^{2}, q / z^{2} ; q^{2}\right)_{\infty}
$$

By this identity, with $q$ replaced by $-q^{5}$ and $z$ replaced by $q^{2}$, we get

$$
\begin{aligned}
\sum_{n=-\infty}^{\infty}(-1)^{n(3 n-1) / 2}\left(q^{n(15 n+7) / 2}+q^{(3 n-2)(5 n-1) / 2}\right) \\
=\left(q^{2},-q^{3},-q^{5} ;-q^{5}\right)_{\infty} \cdot\left(-q,-q^{9} ; q^{10}\right)_{\infty} \\
=\left(q^{2},-q^{3},-q^{5} ;-q^{5}\right)_{\infty} \cdot \frac{\left(q^{2}, q^{18} ; q^{20}\right)_{\infty}}{\left(q, q^{9} ; q^{10}\right)_{\infty}} \\
=\left(-q^{3},-q^{5},-q^{7} ; q^{10}\right)_{\infty} \cdot\left(q^{2}, q^{8}, q^{10} ; q^{10}\right)_{\infty} \cdot \frac{\left(q^{2}, q^{18} ; q^{20}\right)_{\infty}}{\left(q, q^{9} ; q^{10}\right)_{\infty}} \\
=\frac{\left(q^{6}, q^{10}, q^{14} ; q^{20}\right)_{\infty}}{\left(q^{3}, q^{5}, q^{7} ; q^{10}\right)_{\infty}} \cdot \frac{\left(q^{2}, q^{4}, q^{6}, q^{8}, q^{10} ; q^{10}\right)_{\infty}}{\left(q^{4}, q^{6} ; q^{10}\right)_{\infty}} \cdot \frac{\left(q^{2}, q^{18} ; q^{20}\right)_{\infty}}{\left(q, q^{9} ; q^{10}\right)_{\infty}} \\
=\frac{\left(q^{2}, q^{6}, q^{10}, q^{14}, q^{18} ; q^{20}\right)_{\infty}}{\left(q, q^{3}, q^{5}, q^{7}, q^{9} ; q^{10}\right)_{\infty}} \cdot \frac{\left(q^{2} ; q^{2}\right)_{\infty}}{\left(q^{4}, q^{6} ; q^{10}\right)_{\infty}} \\
=\frac{\left(q^{2} ; q^{4}\right)_{\infty}}{\left(q ; q^{2}\right)_{\infty}} \cdot \frac{\left(q^{2} ; q^{2}\right)_{\infty}}{\left(q^{4}, q^{6} ; q^{10}\right)_{\infty}} \\
=\frac{1}{\left(q ; q^{2}\right)_{\infty}\left(q^{4}, q^{6} ; q^{10}\right)_{\infty}} \cdot \frac{\left(q^{2} ; q^{2}\right)_{\infty}}{\left(-q^{2} ; q^{2}\right)_{\infty}} .
\end{aligned}
$$


In a similar way, letting $q \rightarrow q^{5}$ and setting $z=-q$ in (1), we obtain

$$
\begin{gathered}
\sum_{n=-\infty}^{\infty}(-1)^{n(n-1) / 2}\left(q^{n(15 n+1) / 2}+q^{(3 n-1)(5 n-2) / 2}\right) \\
=\frac{1}{\left(q ; q^{2}\right)_{\infty}\left(q^{2}, q^{8} ; q^{10}\right)_{\infty}} \cdot \frac{\left(q^{2} ; q^{2}\right)_{\infty}}{\left(-q^{2} ; q^{2}\right)_{\infty}} .
\end{gathered}
$$

Thus, for $s \in\{2,4\}$, we deduce that

$$
\begin{gathered}
\sum_{n=-\infty}^{\infty}(-1)^{n((s-1) n-1) / 2}\left(q^{n(15 n+3 s-5) / 2}+q^{(3 n-s / 2)(5 n-3+s / 2) / 2}\right) \\
=\frac{1}{\left(q ; q^{2}\right)_{\infty}\left(q^{s}, q^{10-s} ; q^{10}\right)_{\infty}} \cdot \frac{\left(q^{2} ; q^{2}\right)_{\infty}}{\left(-q^{2} ; q^{2}\right)_{\infty}} .
\end{gathered}
$$

On the other hand, the Jacobi triple product identity says that (cf. [7] Eq. (1.6.1)])

$$
\sum_{n=-\infty}^{\infty}(-z)^{n} q^{n(n-1) / 2}=(z, q / z, q ; q)_{\infty}
$$

By this identity, with $q$ replaced by $-q^{5}$ and $z$ replaced by $-q$, we derive

$$
\begin{aligned}
\sum_{n=-\infty}^{\infty}(-1)^{n(n-1) / 2} q^{n(5 n-3) / 2} \\
=\left(-q, q^{4},-q^{5} ;-q^{5}\right)_{\infty} \\
=\left(q^{4}, q^{6}, q^{10} ; q^{10}\right)_{\infty}\left(-q,-q^{5},-q^{9} ; q^{10}\right)_{\infty} \\
=\left(q^{4}, q^{6}, q^{10} ; q^{10}\right)_{\infty} \frac{\left(q^{2}, q^{10}, q^{18} ; q^{20}\right)_{\infty}}{\left(q, q^{5}, q^{9} ; q^{10}\right)_{\infty}} \\
=\frac{\left(q^{2}, q^{4}, q^{6}, q^{8}, q^{10} ; q^{10}\right)_{\infty}}{\left(q, q^{2}, q^{5}, q^{8}, q^{9} ; q^{10}\right)_{\infty}} \cdot \frac{\left(q^{2}, q^{6}, q^{10}, q^{14}, q^{18} ; q^{20}\right)_{\infty}}{\left(q^{6}, q^{14} ; q^{20}\right)_{\infty}} \\
=\frac{\left(q^{2} ; q^{2}\right)_{\infty}}{\left(q, q^{2}, q^{5}, q^{8}, q^{9} ; q^{10}\right)_{\infty}} \cdot \frac{\left(q^{2} ; q^{4}\right)_{\infty}}{\left(q^{6}, q^{14} ; q^{20}\right)_{\infty}} \\
=\frac{\left(q^{3}, q^{4}, q^{6}, q^{7}, q^{10} ; q^{10}\right)_{\infty}}{(q ; q)_{\infty}} \cdot \frac{\left(q^{2}, q^{2}, q^{4} ; q^{4}\right)_{\infty}}{\left(q^{6}, q^{14} ; q^{20}\right)_{\infty}} \\
=\frac{\left(q^{3}, q^{4}, q^{7}, q^{10}, q^{13}, q^{16}, q^{17}, q^{20} ; q^{20}\right)_{\infty}}{(q ; q)_{\infty}} \cdot\left(q^{2}, q^{2}, q^{4} ; q^{4}\right)_{\infty} \\
=\frac{\left(q^{3}, q^{7}, q^{10} ; q^{10}\right)_{\infty}\left(q^{4}, q^{16} ; q^{20}\right)_{\infty}}{(q ; q)_{\infty}} \cdot \frac{\left(q^{2} ; q^{2}\right)_{\infty}}{\left(-q^{2} ; q^{2}\right)_{\infty}} .
\end{aligned}
$$

In a similar way, letting $q \rightarrow-q^{5}$ and setting $z=-q^{3}$ in (3), we obtain

$$
\sum_{n=-\infty}^{\infty}(-1)^{n(n-1) / 2} q^{n(5 n+1) / 2}=\frac{\left(q, q^{9}, q^{10} ; q^{10}\right)_{\infty}\left(q^{8}, q^{12} ; q^{20}\right)_{\infty}}{(q ; q)_{\infty}} \cdot \frac{\left(q^{2} ; q^{2}\right)_{\infty}}{\left(-q^{2} ; q^{2}\right)_{\infty}} .
$$


Thus, for $s \in\{1,3\}$, we deduce that

$$
\begin{aligned}
& \sum_{n=-\infty}^{\infty}(-1)^{n(n+s) / 2} q^{n(5 n-s) / 2} \\
& \quad=\frac{\left(q^{2}, q^{10-s}, q^{10} ; q^{10}\right)_{\infty}\left(q^{10-2 s}, q^{10+2 s} ; q^{20}\right)_{\infty}}{(q ; q)_{\infty}} \cdot \frac{\left(q^{2} ; q^{2}\right)_{\infty}}{\left(-q^{2} ; q^{2}\right)_{\infty}} .
\end{aligned}
$$

The following theta identity is often attributed to Gauss [2, p. 23, eqs. $(2.2 .12)]$ :

$$
1+2 \sum_{n=1}^{\infty}(-1)^{n} q^{n^{2}}=\frac{(q ; q)_{\infty}}{(-q ; q)_{\infty}} .
$$

In 4], the authors considered this theta identity and proved the following truncated form:

$$
\begin{aligned}
& \frac{(-q ; q)_{\infty}}{(q ; q)_{\infty}}\left(1+2 \sum_{j=1}^{k}(-1)^{j} q^{j^{2}}\right) \\
& \quad=1+2(-1)^{k} \frac{(-q ; q)_{k}}{(q ; q)_{k}} \sum_{j=0}^{\infty} \frac{q^{(k+1)(k+j+1)}\left(-q^{k+j+2} ; q\right)_{\infty}}{\left(1-q^{k+j+1}\right)\left(q^{k+j+2} ; q\right)_{\infty}} .
\end{aligned}
$$

By this identity, with $q$ replaced by $q^{2}$, we obtain

$$
\begin{aligned}
& (-1)^{k}\left(\frac{\left(-q^{2} ; q^{2}\right)_{\infty}}{\left(q^{2} ; q^{2}\right)_{\infty}}\left(1+2 \sum_{j=1}^{k}(-1)^{j} q^{2 j^{2}}\right)-1\right) \\
& \quad=2 \frac{\left(-q^{2} ; q^{2}\right)_{k}}{\left(q^{2} ; q^{2}\right)_{k}} \sum_{j=0}^{\infty} \frac{q^{2(k+1)(k+j+1)}\left(-q^{2(k+j+2)} ; q^{2}\right)_{\infty}}{\left(1-q^{2(k+j+1)}\right)\left(q^{2(k+j+2)} ; q^{2}\right)_{\infty}} .
\end{aligned}
$$

Multiplying both sides of this identity by (2), we obtain

$$
\begin{aligned}
(1+ & \left.2 \sum_{n=1}^{k}(-1)^{n} q^{2 n^{2}}\right) \sum_{n=0}^{\infty} \frac{(-q ; q)_{n} q^{n(3 n+s-1) / 2}}{(q ; q)_{2 n+1}} \\
& -\sum_{n=-\infty}^{\infty}(-1)^{n((s-1) n-1) / 2}\left(q^{n(15 n+3 s-5) / 2}+q^{(3 n-s / 2)(5 n-3+s / 2) / 2}\right) \\
=2( & -1)^{k} \frac{\left(-q^{2} ; q^{2}\right)_{k}}{\left(q^{2} ; q^{2}\right)_{k}} \sum_{n=k+1}^{\infty} \frac{q^{2 n(k+1)}\left(-q^{2(n+1)} ; q^{2}\right)_{\infty}}{\left(1-q^{2 n}\right)\left(q^{2(n+1)} ; q^{2}\right)_{\infty}} \times \\
& \times \sum_{n=-\infty}^{\infty}(-1)^{n((s-1) n-1) / 2}\left(q^{n(15 n+3 s-5) / 2}+q^{(3 n-s / 2)(5 n-3+s / 2) / 2}\right) .
\end{aligned}
$$

For each $s \in\{2,4\}$, it is elementary to see that squares that are congruent to $(3 s-5)^{2}$ modulo 120 are of the form $n(15 n+3 s-5) / 2$ or $(3 n-s / 2)(5 n-3+s / 2) / 2$ with $k \in \mathbb{Z}$. The first identity is proved. 
In a similar way, multiplying both sides of (5) by (4), we obtain

$$
\begin{aligned}
& \left(1+2 \sum_{n=1}^{k}(-1)^{n} q^{2 n^{2}}\right) \sum_{n=0}^{\infty} \frac{q^{n(n+1)}}{(q ; q)_{2 n+(s-1) / 2}}-\sum_{n=-\infty}^{\infty}(-1)^{n(n+s) / 2} q^{n(5 n-s) / 2} \\
& =2(-1)^{k} \frac{\left(-q^{2} ; q^{2}\right)_{k}}{\left(q^{2} ; q^{2}\right)_{k}} \sum_{n=k+1}^{\infty} \frac{q^{2 n(k+1)}\left(-q^{2(n+1)} ; q^{2}\right)_{\infty}}{\left(1-q^{2 n}\right)\left(q^{2(n+1)} ; q^{2}\right)_{\infty}} \sum_{n=-\infty}^{\infty}(-1)^{n(n+s) / 2} q^{n(5 n-s) / 2}
\end{aligned}
$$

For each $s \in\{1,3\}$, it is elementary to see that squares that are congruent to $s^{2}$ modulo 40 are of the form $n(5 n-s) / 2$ with $n \in \mathbb{Z}$. Thus we deduce the second identity.

\section{Proof of Corollary 1.2}

For each $s \in\{2,4\}$, the generating function for $R_{s}(n)$ can be written as follows:

$$
\sum_{n=0}^{\infty} R_{s}(n) q^{n}=\frac{\left(q^{6-s}, q^{4+s}, q^{10} ; q^{10}\right)_{\infty}}{(q ; q)_{\infty}} .
$$

By the Jacobi triple product identity (3), with $q$ replaced by $q^{10}$ and $z$ replaced by $q^{6-s}$, we get

$$
\sum_{n=-\infty}^{\infty}(-1)^{n} q^{n(5 n+1-s)}=\left(q^{6-s}, q^{4+s}, q^{10} ; q^{10}\right)_{\infty}
$$

Thus we obtain

$$
\begin{aligned}
\sum_{n=0}^{\infty} R_{s}(n) q^{n} & =\left(\sum_{n=0}^{\infty} p(n) q^{n}\right)\left(\sum_{n=-\infty}^{\infty}(-1)^{n} q^{n(5 n+1-s)}\right) \\
& =\sum_{n=0}^{\infty}\left(\sum_{k=-\infty}^{\infty}(-1)^{k} p(n-k(5 k+1-s))\right) q^{n} .
\end{aligned}
$$

Equating the coefficient of $q^{n}$ in this equation, we obtain the following decomposition of $R_{s}(n)$ in terms of the partition function $p(n)$ :

$$
R_{s}(n)=\sum_{k=-\infty}^{\infty}(-1)^{k} p(n-k(5 k+1-s)) .
$$

It is an easy exercise to show that the squares congruent to $(s-1)^{2}$ modulo 20 are of the form $k(5 k+1-s)$ with $k \in \mathbb{Z}$.

For each $s \in\{1,3\}$, considering Watson's quintuple product identity (10), with $q$ replaced by $q^{10}$ and $z$ replaced by $q^{s}$, we get

$$
\sum_{n=0}^{\infty} R_{s}^{*}(n) q^{n}=\frac{1}{(q ; q)_{\infty}} \sum_{n=-\infty}^{\infty} q^{15 n^{2}-(5-3 s) n}\left(1-q^{10 n+s}\right)
$$




$$
=\left(\sum_{n=0}^{\infty} p(n) q^{n}\right)\left(\sum_{n=-\infty}^{\infty}\left(q^{15 n^{2}-(5-3 s) n}-q^{15 n^{2}+(5+3 s) n+s}\right)\right) .
$$

Equating the coefficient of $q^{n}$ in this equation, we obtain

$R_{s}^{*}(n)=\sum_{k=-\infty}^{\infty}\left(p\left(n-\left(15 k^{2}-(5-3 s) k\right)\right)-p\left(n-\left(15 k^{2}+(5+3 s) k+s\right)\right)\right)$.

It is an easy exercise to show that the squares congruent to $(s+1)^{2} / 4$ modulo 15 are of the form $15 k^{2}-(5-3 s) k$ or $15 k^{2}+(5+3 s) k+s$ with $k \in \mathbb{Z}$.

\section{Open problems}

Related to relations (6) and (7), we remark that there is a substantial amount of numerical evidence to state the following conjecture.

Conjecture 1. Let $k$ be a positive integer.

1. For $s \in\{2,4\}$,

$$
\begin{aligned}
(1+ & \left.2 \sum_{n=1}^{k}(-1)^{n} q^{2 n^{2}}\right) \sum_{n=0}^{\infty} \frac{(-q ; q)_{n} q^{n(3 n+s-1) / 2}}{(q ; q)_{2 n+1}} \\
& -\sum_{n=-\infty}^{\infty}(-1)^{n((s-1) n-1) / 2}\left(q^{n(15 n+3 s-5) / 2}+q^{(3 n-s / 2)(5 n-3+s / 2) / 2}\right)
\end{aligned}
$$

has nonnegative coefficients if $k$ is even and nonpositive coefficients if $k$ is odd.

2. For $s \in\{1,3\}$,

$$
\left(1+2 \sum_{n=1}^{k}(-1)^{n} q^{2 n^{2}}\right) \sum_{n=0}^{\infty} \frac{q^{n(n+1)}}{(q ; q)_{2 n+(s-1) / 2}}-\sum_{n=-\infty}^{\infty}(-1)^{n(n+s) / 2} q^{n(5 n-s) / 2}
$$

has nonnegative coefficients if $k$ is even and nonpositive coefficients if $k$ is odd.

Assuming this conjecture, we derive the following families of linear inequalities involving $R_{s}(n)$ and $R_{s}^{*}(n)$.

Conjecture 2. Let $k$ be a positive integer.

1. For $s \in\{2,4\}$,

$$
(-1)^{k}\left(R_{s}(n)+2 \sum_{j=1}^{k}(-1)^{k} R_{s}\left(n-2 j^{2}\right)-\rho_{s}(n)\right) \geqslant 0,
$$


where

$$
\rho_{s}(n)=\left\{\begin{aligned}
(-1)^{m((s-1) m-1) / 2}, & \text { if } n=m(15 m+3 s-5) / 2 \text { or } \\
n & =(3 m-s / 2)(5 m-3+s / 2) / 2, m \in \mathbb{Z}, \\
0, & \text { otherwise. }
\end{aligned}\right.
$$

2. For $s \in\{1,3\}$,

$$
(-1)^{k}\left(R_{s}^{*}(n)+2 \sum_{j=1}^{k} R_{s}^{*}\left(n-2 j^{2}\right)-\rho_{s}^{*}(n)\right) \geqslant 0,
$$

where

$$
\rho_{s}^{*}(n)= \begin{cases}(-1)^{m(m+s) / 2}, & \text { if } n=m(5 m-s) / 2, m \in \mathbb{Z}, \\ 0, & \text { otherwise. }\end{cases}
$$

It would be very appealing to obtain other $q$-series congruences that involve other statistical mechanics partition functions appearing in Baxter's solution of the hard-hexagon model.

\section{References}

[1] G.E. Andrews, The hard-hexagon model and Rogers-Ramanujan type identities. Proc. Natl Acad. Sci. USA, 78(9) (1981) 5290-5292.

[2] G.E. Andrews, The Theory of Partitions, Cambridge Mathematical Library, Cambridge University Press, Cambridge, 1998. Reprint of the 1976 original.

[3] G.E. Andrews, Euler's "De Partitio Numerorum", Bull. Amer. Math. Soc. (N.S.) 44 (2007) 561-573.

[4] G.E. Andrews and M. Merca, Truncated theta series and a problem of Guo and Zeng, J. Combin. Theory Ser. A, 154 (2018) 610-619.

[5] L. Carlitz and M.V. Subbarao, A simple proof of the quintuple product identity, Proc. Amer. Math. Soc. 32 (1972), 42-44.

[6] C. Ballantine and M. Merca, Parity of sums of partition numbers and squares in arithmetic progressions. Ramanujan $J$ 44 617-630 (2017).

[7] G. Gasper and M. Rahman, Basic hypergeometric series, Encyclopedia of Mathematics And Its Applications 35, Cambridge University Press, Cambridge, 1990.

[8] M. Merca, Fast algorithm for generating ascending compositions, J. Math. Model. Algorithms 11 (2012) 89-104. 
[9] M. Merca, Binary diagrams for storing ascending compositions, Comput. J. 56(11) (2013) 1320-1327.

[10] M.V. Subbarao and M. Vidyasagar, On Watson's quintuple product identity, Proc. Amer. Math. Soc. 26 (1970), 23-27. 